\title{
Erratum to: UPLC versus HPLC on Drug Analysis: Advantageous, Applications and Their Validation Parameters
}

Mehmet Gumustas • Sevinc Kurbanoglu •

Bengi Uslu $\cdot$ Sibel A. Ozkan

Published online: 28 July 2013

(c) Springer-Verlag Berlin Heidelberg 2013

\section{Erratum to: Chromatographia}

DOI 10.1007/s10337-013-2477-8

The authors would like to call the reader's attention to the fact that unfortunately there were several references faultily assigned in Table 1 . Please find the corrected part of Table 1 below:

The online version of the original article can be found under doi:10.1007/s10337-013-2477-8.

M. Gumustas · S. Kurbanoglu · B. Uslu · S. A. Ozkan ( $₫)$ Department of Analytical Chemistry, Faculty of Pharmacy, Ankara University, Ankara, Turkey

e-mail: ozkan@pharmacy.ankara.edu.tr

M. Gumustas

Department of Chemistry, Science and Literature Faculty,

Hitit University, Corum, Turkey 
Table 1 Some selected examples of HPLC applications on drug analysis

\begin{tabular}{|c|c|c|c|c|c|c|c|}
\hline \multirow[t]{2}{*}{ Compounds } & \multicolumn{5}{|l|}{ Validation } & \multirow{2}{*}{$\begin{array}{l}\text { Applied } \\
\text { sample }\end{array}$} & \multirow[t]{2}{*}{ Ref. } \\
\hline & $\begin{array}{l}\text { Linearity range } \\
(\mu \mathrm{g} / \mathrm{mL})\end{array}$ & $\begin{array}{l}\text { LOD } \\
(\mu \mathrm{g} / \mathrm{mL})\end{array}$ & $\begin{array}{l}\text { LOQ } \\
(\mu \mathrm{g} / \mathrm{mL})\end{array}$ & $\begin{array}{l}\text { Precision } \\
(\text { RSD \%) }\end{array}$ & $\begin{array}{l}\text { Accuracy } \\
(\%)\end{array}$ & & \\
\hline Theophylline & $0.5-40$ & 0.30 & 0.40 & 0.26 & 99.0 & PP & [132] \\
\hline Guaiphenesin & $1.5-45$ & 0.40 & 1.20 & 0.72 & 99.8 & & \\
\hline Ambroxol hydrochloride & $1-80$ & $\begin{array}{l}0.40 \\
\mathrm{mg} / \mathrm{kg}\end{array}$ & $\begin{array}{l}0.60 \\
\mathrm{mg} / \mathrm{kg}\end{array}$ & 0.47 & 99.9 & & \\
\hline Histamine & $\begin{array}{l}5.0-100 \\
\mathrm{ng} / \mathrm{g}\end{array}$ & $\begin{array}{l}1.5 \\
\mathrm{ng} / \mathrm{g}\end{array}$ & $\begin{array}{l}4.5 \\
\mathrm{ng} / \mathrm{g}\end{array}$ & 1.35 & 95.77 & Tuna fish & [133] \\
\hline Oxytetracycline & $50-5,000$ & 4.4 & 10 & 3.45 & 92.1 & Chicken meat & [134] \\
\hline Tetracycline & & 5 & 13 & 4.08 & 71.88 & & \\
\hline Chlortetracycline & & 10 & 27 & 2.33 & 84.88 & & \\
\hline Doxycycline & & 7 & 24 & 5.44 & 90.0 & & \\
\hline & $\mathrm{ng} / \mathrm{g}$ & $\mathrm{ng} / \mathrm{g}$ & $\mathrm{ng} / \mathrm{g}$ & & & & \\
\hline Oxytetracycline & $50-5,000$ & 4.4 & 10 & 7.94 & 82.22 & Chicken liver & [134] \\
\hline Tetracycline & & 5 & 13 & 6.42 & 68.66 & & \\
\hline Chlortetracycline & & 10 & 27 & 8.08 & 81.05 & & \\
\hline Doxycycline & & 7 & 24 & 5.07 & 76.66 & & \\
\hline Coumarin & $0.1-40$ & 0.0132 & 0.0417 & 1.21 & 99.83 & Plant & [135] \\
\hline 2-Hydroxyl cinnamaldehyde & $0.1-10$ & 0.0205 & 0.0698 & 0.60 & 98.27 & & \\
\hline Cinnamyl alcohol & $0.2-10$ & 0.0433 & 0.1294 & 0.41 & 100.14 & & \\
\hline Cinnamic acid & $0.1-40$ & 0.0092 & 0.0173 & 1.83 & 98.84 & & \\
\hline Cinnamaldehyde & $1.0-400$ & 0.0165 & 0.0533 & 1.36 & 100.13 & & \\
\hline 2-Ethoxy cinnamaldehyde & $0.5-5.0$ & 0.0916 & 0.3042 & 0.65 & 101.70 & & \\
\hline Eugenol & $0.1-5.0$ & 0.0183 & 0.0501 & 1.28 & 100.44 & & \\
\hline & $\mu \mathrm{g} / \mathrm{g}$ & $\mu \mathrm{g} / \mathrm{g}$ & $\mu \mathrm{g} / \mathrm{g}$ & & & & \\
\hline Albendazole & $0.1-2.0$ & 0.016 & 0.100 & 11.1 & $82.1-77.4$ & Egg & [136] \\
\hline Albendazole sulphoxide & & 0.064 & 0.250 & 10.7 & $88.8-80.1$ & & \\
\hline Albendazole sulphone & & 0.072 & 0.250 & 8.90 & $89.8-84.8$ & & \\
\hline A-Albendazole sulphone & & 0.030 & 0.100 & 7.80 & $96.5-85.6$ & & \\
\hline Fenbendazole & & 0.009 & 0.100 & 18.0 & $83.0-68.8$ & & \\
\hline Fenbendazole sulphoxide & & 0.134 & 0.250 & 5.70 & $89.7-82.0$ & & \\
\hline Fenbendazole sulphone & & 0.070 & 0.250 & 6.00 & $98.3-89.3$ & & \\
\hline Flubendazole & & 0.029 & 0.100 & 10.0 & $90.7-68.9$ & & \\
\hline Hydrolysed flubendazole & & 0.061 & 0.250 & 5.80 & $92.0-78.9$ & & \\
\hline Reduced flubendazole & & 0.005 & 0.100 & 8.90 & $86.9-81.0$ & & \\
\hline Albendazole & $0.05-2.0$ & 0.007 & 0.050 & 8.50 & $98.2-89.4$ & Plasma & [136] \\
\hline Albendazole sulphoxide & & 0.087 & 0.125 & 0.518 & $86.2-81.8$ & & \\
\hline Albendazole sulphone & & 0.009 & 0.050 & 7.21 & $93.4-90.4$ & & \\
\hline A-Albendazole sulphone & & 0.012 & 0.050 & 3.83 & $95.5-88.9$ & & \\
\hline Fenbendazole & & 0.017 & 0.050 & 6.42 & $82.1-71.4$ & & \\
\hline Fenbendazole sulphoxide & & 0.042 & 0.125 & 13.4 & $92.9-87.0$ & & \\
\hline Fenbendazole sulphone & & 0.024 & 0.125 & 6.95 & $99.4-94.1$ & & \\
\hline Flubendazole & & 0.014 & 0.050 & 9.88 & $91.9-85.9$ & & \\
\hline Hydrolysed flubendazole & & 0.004 & 0.050 & 6.85 & $111.0-92.4$ & & \\
\hline Reduced flubendazole & & 0.007 & 0.050 & 7.89 & $93.7-82.8$ & & \\
\hline Tetrandrine & $0.051-5.088$ & 0.010 & 0.033 & $<10$ & 94.33 & Rabbit plasma & [137] \\
\hline Telmisartan & $1.0-10.0$ & 0.054 & 0.180 & $<3.60$ & 89.0 & Human plasma & [138] \\
\hline Hydrochlorothiazide & $0.31-3.12$ & 0.043 & 0.140 & & 95.4 & & \\
\hline
\end{tabular}


Table 1 continued

\begin{tabular}{|c|c|c|c|c|c|c|c|}
\hline \multirow[t]{2}{*}{ Compounds } & \multicolumn{5}{|l|}{ Validation } & \multirow{2}{*}{$\begin{array}{l}\text { Applied } \\
\text { sample }\end{array}$} & \multirow[t]{2}{*}{ Ref. } \\
\hline & $\begin{array}{l}\text { Linearity range } \\
(\mu \mathrm{g} / \mathrm{mL})\end{array}$ & $\begin{array}{l}\text { LOD } \\
(\mu \mathrm{g} / \mathrm{mL})\end{array}$ & $\begin{array}{l}\text { LOQ } \\
(\mu \mathrm{g} / \mathrm{mL})\end{array}$ & $\begin{array}{l}\text { Precision } \\
\text { (RSD \%) }\end{array}$ & $\begin{array}{l}\text { Accuracy } \\
(\%)\end{array}$ & & \\
\hline Thiocolchicoside & $40.48-121.4$ & - & - & 0.29 & 100.25 & PP & [139] \\
\hline Diclofenac potassium & $24.91-74.72$ & & & 1.31 & 99.80 & & \\
\hline Ceftazidime & $1.0-16.0$ & 0.361 & 1.202 & 0.263 & 99.95 & PP & [140] \\
\hline Ceftizoxime & $1.0-20.0$ & 0.234 & 0.780 & 0.187 & 100.10 & & \\
\hline Cefdinir & $0.5-16.0$ & 0.0457 & 0.139 & 0.002 & $\sqrt{ }$ & PP & [141] \\
\hline Cefixime & $0.5-16.0$ & 0.0268 & 0.081 & 0.021 & & & \\
\hline Phenylephrine & $5-30$ & 0.877 & 2.658 & 0.62 & 100.56 & PP & [142] \\
\hline Paracetamol & $100-600$ & 27.75 & 84.09 & 0.88 & 100.31 & & \\
\hline Gemifloxacin & $0.25-20$ & 0.004 & 0.013 & 0.069 & 99.96 & $\mathrm{PP}$ & [143] \\
\hline Granisetron & $0.25-15$ & 0.006 & 0.021 & 0.036 & 100.16 & $\mathrm{PP}$ & [144] \\
\hline Mitoxantrone & $0.005-1$ & - & 0.005 & 2.8 & 98.2 & Mouse plasma & [145] \\
\hline Fexofenadine & $0.3-50$ & 0.19 & 5.00 & 2.11 & 97.9 & Human serum & [146] \\
\hline Levocetirizine & & 0.16 & 0.55 & 0.90 & 99.4 & & \\
\hline Buclizine & & 0.09 & 0.32 & 0.49 & 102.0 & & \\
\hline Gliquidone & & 0.10 & 0.33 & 0.49 & 102.0 & & \\
\hline
\end{tabular}

$D A D$ diode array detector, $F L D$ fluorescence detector, $C A D$ charged aerosol detector, $T F A$ trifluoroacetic acid, TEA triethylamine, $A C N$ acetonitrile, $M e O H$ methanol, $R I$ refractive index, $P P$ pharmaceutical preparation, $T P G S$ tocopherol polyethylene glycol succinate, HFBA heptafluorobutyric acid 